\title{
SLIP STACKING EXPERIMENTS AT FERMILAB MAIN INJECTOR
}

\author{
K. Koba, T. Berenc, B. Barnes, B. Chase, I. Kourbanis, J. MacLachlan, K. Meisner, J. Steimel \\ FNAL, Batavia, IL 60510, USA
}

\begin{abstract}
In order to achieve an increase in proton intensity, Fermilab Main Injector will use a stacking process called "slip stacking"[1]. The intensity will be doubled by injecting one train of bunches at a slightly lower energy, another at a slightly higher energy, then bringing them together for the final capture. Beam studies have started for this process and we have already verified that, at least for a low beam intensity, the stacking procedure works as expected[2][3][4]. For high intensity operation, development work of the feedback and feedforward systems is under way[5][6][7].
\end{abstract}

\section{INTRODUCTION}

The Fermilab Main Injector (MI) accelerates protons and extracts them to a target to produce antiprotons. In the operation cycle for the antiproton production, 84 bunches are injected from Booster to MI. Bunches are accelerated from $8 \mathrm{GeV}$ to $120 \mathrm{GeV}$ and extracted to hit the production target. The total beam intensity is $4.5^{*} 10^{12}$ particles per pulse (ppp) with a cycle of $1.5 \mathrm{sec}$. The Main Injector parameters are listed in Table 1.

Run IIb will follow Run IIa and has a luminosity goal $4.1 * 10^{32} \mathrm{~cm}^{-2} \mathrm{sec}^{-1}$ requiring $9.634 * 10^{12}$ total antiprotons in the Tevatron. In order to achieve this goal, we need $9.0 * 10^{12}$ protons in 84 bunches in the Main Injector for antiproton production. Higher proton intensities are required to get more antiprotons and higher stacking rates in the accumulator. In order to achieve this, we are planning to use a scheme called "slip stacking". With slip stacking, the intensity of the bunches can be doubled by injecting one bunch train at slightly lower energy, another train at slightly higher energy and bringing them together.

Beam studies of the slip stacking process have started and we have already established that the stacking procedure works as expected.

Table 1: Main Injector parameters on stacking cycle

\begin{tabular}{|l|l|}
\hline RF frequency @ injection & $52.811400 \mathrm{~Hz}$ \\
\hline Proton energy @ injection & $8 \mathrm{GeV}$ \\
\hline Proton energy @ extraction & $120 \mathrm{GeV}$ \\
\hline Harmonic number & 588 \\
\hline Transition gamma & 21.6 \\
\hline Mean radius & $528.3 \mathrm{~m}$ \\
\hline
\end{tabular}

\footnotetext{
* Work supported by the Universities Research Assoc., Inc., under contract DE-AC02-76CH03000 with the U.S. Dept. of Energy.
}

\section{RF PARAMETERS}

For slip stacking, we use three different radio frequency (RF) systems and follow four steps. Step 1: The first bunch train is injected from the Booster on the central orbit and captured by the first RF system. To make a room for the second bunch train, the first bunch train is then decelerated until it circulates on the inside of the central orbit. Step 2: The second bunch train is injected on the central orbit and captured by the second RF system. Step 3: As the two bunch trains have slightly different energies, they can move relative to each other. Both bunch trains are accelerated till they are separated by the same amount from the central energy. Step 4: When the two bunch trains coincide at the same longitudinal location, they are captured by the third RF system.

Since two bunch trains have different energies, MI must have an enough momentum aperture to accept both. The momentum aperture of $\mathrm{MI}$ is $+/-0.7 \%$ at injection, corresponding to the frequency separation of $+/-3000 \mathrm{~Hz}$ from the central value of $52.8114 \mathrm{MHz}$.

\section{MI cavities and Injection Matching}

MI has eighteen $53 \mathrm{MHz}$ cavities. These are separated into the two groups, each with 9 cavities. Low level RF (LLRF) signals go to two groups of cavities individually. For the slip stacking, we are using one out of 9 cavities in each group at injection. The RF voltage at injection is adjusted so that one cavity produces $62 \mathrm{kV}$ in order to achieve a low momentum spread.

At extraction, the Booster RF voltage is $380 \mathrm{kV}$, which matches to a MI injection voltage of $1 \mathrm{MV}$ for normal operation. Since the MI injection RF voltage is $62 \mathrm{kV}$ for the slip stacking, a bunch rotation is carried out in Booster before extraction so that the bunch shape will be matched to the RF bucket of MI at injection. As the Booster RF voltage is rapidly reduced, the bunch starts to rotate in the phase space. After a quarter of synchrotron period, the bunch is injected into MI.

\section{Frequency Curve}

The frequency as a function of time is shown in Figure 1 for the first and the second RF systems. The frequency separation was kept at $1200 \mathrm{~Hz}$ which is enough to keep the bunch shape unchanged.

The first bunch train was injected on the central orbit with nominal frequency at $0.13 \mathrm{msec}$ and captured by the first RF system of $62 \mathrm{kV}$. At this time, the frequency of the second RF system was $1200 \mathrm{~Hz}$ higher than the first RF system. The first bunch was then decelerated to a frequency $1200 \mathrm{~Hz}$ lower than the original value. After 
one Booster cycle of $66.7 \mathrm{msec}$, the second train was injected on the central orbit and captured by the second RF system. After slipping, both bunch trains were captured by all 18 cavities with $800 \mathrm{kV}$ and 0 frequency separation.

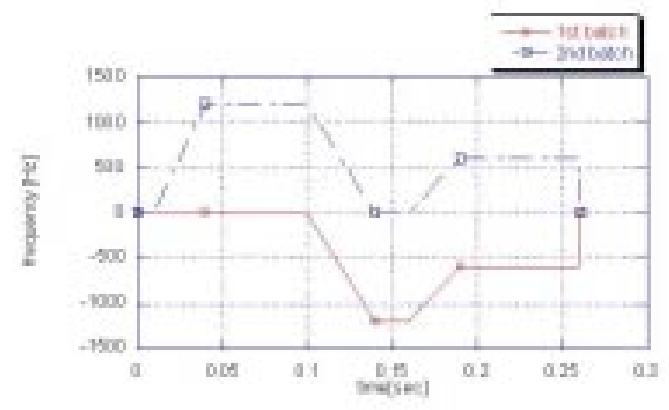

Figure 1: Frequency for first bunch train and second bunch train as a function of time.

\section{PREVIOUS BEAM STUDY}

Two bunch trains, each with 82 bunches, were injected into MI. The total intensity for two trains was low, $0.8 * 10^{12} \mathrm{ppp}$, in order to reduce beam loading effects.

Figure 2, a mountain range picture of the signals from the wall current monitor (WCM), reveals the progress of slip stacking from the beginning to the end. The WCM had a resolution of $0.5 \mathrm{~ns} / \mathrm{sample}$. The data were obtained every $1.42 \mathrm{~ms}$ for $0.18 \mathrm{~s}$. There was no beam loss during the slip stacking process.

The length of the first bunch of the first bunch train shown in Fig. 2 was used to calculate the emittance. Figure 3 shows the calculated $95 \%$ emittance during 0.18 s. Since there was an empty space between upper and lower bunches, there should be an emittance growth. The emittance growth expected from simulation is a factor of 3.2. From the plot, we see that there is no emittance growth before the two batches are recaptured by one RF bucket but there is a factor of 4.0 emittance growth after the recapture.

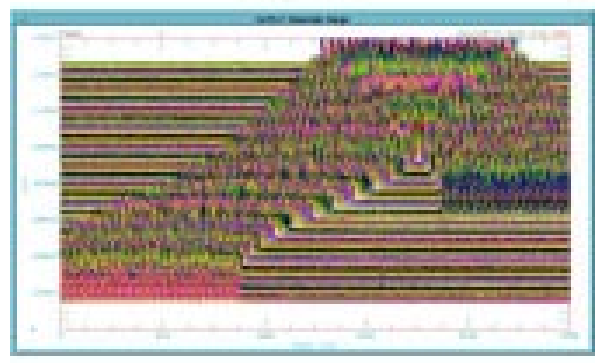

Figure 2: Mt range plot. The signal came from WCM with a resolution of $0.5 \mathrm{~ns} / \mathrm{sample}$. The data were obtained every $1.42 \mathrm{~ms}$ for $0.18 \mathrm{~s}$.

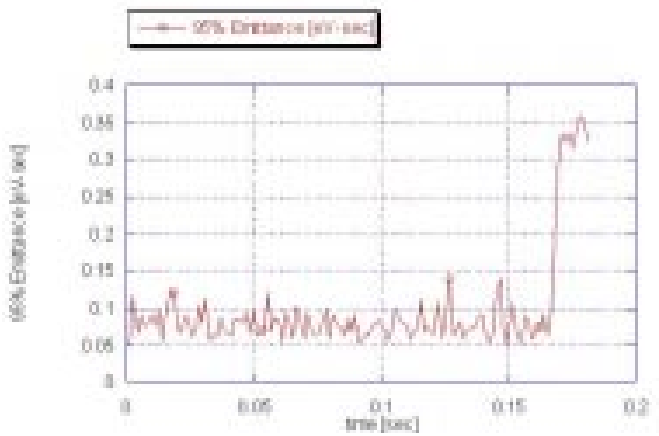

Figure 3: The 95\% emittance calculated by bunch length shown in Fig.2.

\section{SLIP STACKING PHASE VARIATION}

Frequency curves (Fig.1) applied to the beam are calculated assuming that, at the time of recapture, two bunch trains are sitting exactly on the same longitudinal location, that is, two cavities are in phase. However, MI slip stacking studies revealed cycle to cycle variation in the RF system phase slip. This produced an unreliable RF voltage for recapture.

The largest phase variation was traced to timing jitter on the $720 \mathrm{~Hz}$ line locked Tevatron Clock event. A MI LLRF system Digital Signal Processor (DSP) generates the frequency curves from user tuned tables. The frequency curves are a function of time and are integrated by the DSP to apply phase modulation to the RF systems that slip the bunch trains. The DSP processes run at $720 \mathrm{~Hz}, 23 * 720 \mathrm{~Hz}$, and $138 * 720 \mathrm{~Hz}$ via a scheduler that is synchronized with the Tevatron Clock event by a phase lock loop (PLL). As the PLL input jitters and the DSP follows the jitter, the frequency curves output and its integral (RF phase) varies.

The RF phase variation was reduced to $0^{\circ}$ by changing the frequency curve generation from the Tevatron Clock time base to accurate LLRF internal time.

\section{SIMULATION FOR RECAPTURE}

To understand what caused the emittance growth at recapture, simulation studies were carried out using the code ESME[8]. In the simulation studies, the time and voltage to recapture bunches were varied to match the observed RF phase variation.

The delay was changed from 0 to $250 \mu$ sec causing a $+/-60$ degrees phase variation and the $95 \%$ bunch length after $15 \mathrm{msec}$ was measured. The emittance plotted in Fig. 4 was estimated from the bunch length. It increased by a factor of 3.2 to 7.0 with increasing delay. Since the RF phase variation produced errors as large as 45 degrees, it could cause the emittance growth. 
The recapture voltage was changed from 0.5 to $1.0 \mathrm{MV}$ because the voltage is changing from 0.8 to $1.0 \mathrm{MV}$ after the time to recapture. The $95 \%$ emittance plotted in Fig. 5 shows the emittance growth by a factor of 3.2 to 3.6.

By the two simulation studies, it was verified that the emittance growth was mainly due to the RF phase variation produced by the time error of Tevatron Clock event.

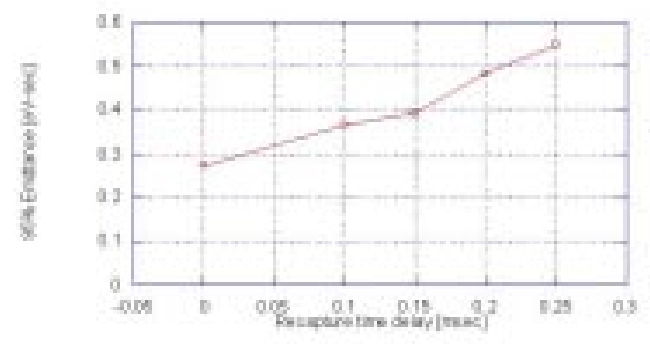

Figure 4: The estimated $95 \%$ emittance vs. recapture delay time.

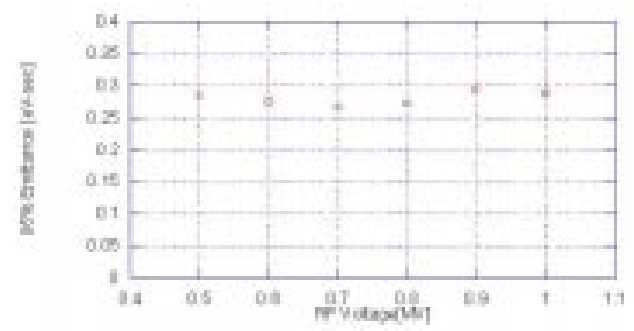

Figure 5: The estimated $95 \%$ emittance vs. recapture RF voltage.

\section{BEAM STUDY}

Previous beam studies have reported that there is an emittance growth when two bunch trains are recaptured. Simulation studies have indicated that the emittance growth is caused by variation in the final RF system phase. The phase variation was eliminated and the $95 \%$ longitudinal emittance was measured again. A factor of 3.2 in the observed emittance growth is the same as simulation results and there is no unexpected emittance growth during slip stacking.

\section{ACCELERATION}

Acceleration was also tried. Figure 6 shows the beam intensity during beam acceleration. Approximately $1.0 * 10^{12}$ total particles were injected, slip stacked and accelerated to $120 \mathrm{GeV}$. There was a beam loss of $\sim 2 \%$ at the beginning of acceleration.

The emittance at $120 \mathrm{GeV}$ was measured. The observed value of $0.24 \mathrm{eV}$-sec is less than the one we currently have in the normal operation cycle.

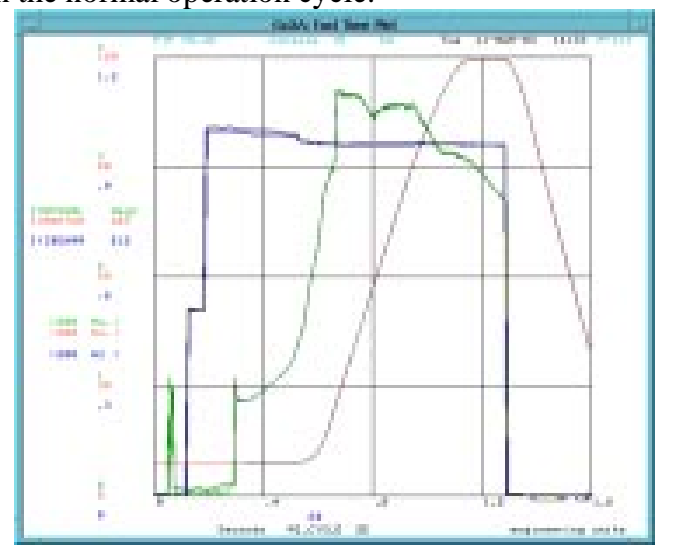

Figure 6: RFSUML: RF voltage[MV], MMNTUM: momentum[GeV/c], IBEMM: total beam intensity [E12 ppp]

\section{CONCLUSION}

Beam studies have stared for the slip stacking process, and we have already verified that, at least for low beam intensity, the stacking procedure works as expected.

There was no beam loss during the process, but there was emittance growth when two bunch trains were recaptured. Simulation studies have indicated that the emittance growth is caused by RF phase variation.

The RF phase variation was eliminated and the emittance was measured again. No undesirable emittance blow up has been observed.

Beam has been accelerated to $120 \mathrm{GeV}$ with a beam loss of $\sim 2 \%$ at the beginning of acceleration.

For higher intensity operation, development of the feedback and feedforward system is under way.

\section{REFERENCES}

[1] J. A. MacLachlan, "The Beam Dynamics of Slip Stacking", Fermilab FN-0711, November 2001.

[2] K. Koba, "Slip Stacking," ICFA2002, April 2002.

[3] K. Koba," Emittance measurement and acceleration Revision of MI-0294”, MI-0294

[4] K. Koba," Slip Stacking at Low Intensity - Status of the Beam Studies", MI-0296

[5] J.Dey, J. Steimel and J. Reid, "Narrowband Beam Loading Compensation in the Fermilab Main Injector Accelerating Cavities", 2001 PAC, p. 876, Chicago, June 2001.

[6] J. Dey, et al, "53 MHz Feedforward Beam Loading Compensation in the Fermilab Main Injector," PAC '03, These proceedings.

[7] J. Steimel et al,“ Beam Loading Compensation for Slip Stacking”, PAC '03, These proceedings.

[8] J. A. MacLachlan, "Users Guide to ESME", 2000. 